\title{
Staphylococcus Aureus Gastroenteritis
}

National Cancer Institute

\section{Source}

National Cancer Institute. Staphylococcus Aureus Gastroenteritis. NCI Thesaurus. Code C35036.

Gastroenteritis resulting from an infection with Staphylococcus Aureus. 